\title{
Rapid screening and early precautions for carbapenem-resistant Acinetobacter baumannii carriers decreased nosocomial transmission in hospital settings: a quasi-experimental study
}

Norihisa Yamamoto ${ }^{1,2}$, Shigeto Hamaguchi ${ }^{1,2}$, Yukihiro Akeda ${ }^{1,2^{*}}$, Pitak Santanirand ${ }^{3}$, Narong Chaihongsa ${ }^{3}$, Suntariya Sirichot ${ }^{3}$, Suwichak Chiaranaicharoen ${ }^{3}$, Hideharu Hagiya ${ }^{1,2}$, Kouji Yamamoto ${ }^{4}$, Anusak Kerdsin ${ }^{5}$, Kazuhisa Okada ${ }^{2}$, Hisao Yoshida', Shigeyuki Hamada ${ }^{2}$, Kazunori Oishi ${ }^{6}$, Kumthorn Malathum $^{3}$ and Kazunori Tomono'

\begin{abstract}
Background: Active surveillance has the potential to prevent nosocomial transmission of carbapenem-resistant Acinetobacter baumannii (CRAB). We assessed whether rapid diagnosis using clinical specimen-direct loop-mediated isothermal amplification (LAMP), a rapid molecular diagnostic assay, and subsequent intervention, could reduce CRAB nosocomial transmission in intensive care units (ICUs).

Methods: A before and after (quasi-experimental) study was conducted in two ICUs at the Mahidol University Faculty of Medicine Ramathibodi Hospital with 3 months of observational period followed by 9 months of interventional period. All patients were screened for CRAB using both the culture and LAMP method from rectal swab and/or bronchial aspirates (intubated patients only) upon admission, weekly thereafter, and upon discharge. During the pre-intervention period, we performed contact precautions based on culture results. In contrast, during the intervention period, we initiated contact precautions within a few hours after sample collection on the basis of LAMP results.

Results: A total of 1335 patients were admitted to the ICUs, of which 866 patients (pre-intervention period: 187; intervention period: 679) were eligible for this study. Incidence rate of CRAB infection decreased to 20.9 per 1000 patient-days in the intervention period from 35.2 in the pre-intervention period $(P<0.02)$. The calculated hazard ratio of CRAB transmission was 0.65 (95\% confidence interval [CI], 0.44-0.97). Risk factors for CRAB acquisition included exposure to carbapenem (hazard ratio, 2.54 [95\% Cl: 1.61-5.57]).

Conclusions: LAMP screening for CRAB upon ICU admission proved feasible for routine clinical practice. Rapid screening using LAMP followed by early intervention may reduce CRAB transmission rates in ICUs when compared to conventional intervention.
\end{abstract}

Keywords: Carbapenem-resistant Acinetobacter baumannii (CRAB), Nosocomial transmission, LAMP (Loop-mediated isothermal amplification), Rapid molecular diagnosis., Rapid intervention, Intensive care unit.

\footnotetext{
* Correspondence: akeda@biken.osaka-u.ac.jp

${ }^{1}$ Department of Infection Control and Prevention, Osaka University Graduate

School of Medicine, 2-2 Yamadaoka, Suita, Osaka 565-0871, Japan

${ }^{2}$ Research Institute for Microbial Diseases, Osaka University, Osaka, Japan

Full list of author information is available at the end of the article
}

(c) The Author(s). 2019 Open Access This article is distributed under the terms of the Creative Commons Attribution 4.0 International License (http://creativecommons.org/licenses/by/4.0/), which permits unrestricted use, distribution, and reproduction in any medium, provided you give appropriate credit to the original author(s) and the source, provide a link to the Creative Commons license, and indicate if changes were made. The Creative Commons Public Domain Dedication waiver (http://creativecommons.org/publicdomain/zero/1.0/) applies to the data made available in this article, unless otherwise stated. 


\section{Background}

Acinetobacter baumannii is an important opportunistic pathogen that causes a variety of nosocomial infections, especially in an intensive care unit (ICU) [1-4]. The organism frequently exhibits resistance to most commercially available antibiotics [1]. In particular, carbapenem-resistant Acinetobacter baumannii (CRAB) infections present limited therapeutic options and are associated with high morbidity and mortality as well as longer hospitalization times [3-6]. Patients colonized with CRAB have a greater risk of developing active $C R A B$ infections as well as initiating nosocomial transmission $[5,6]$. These risks from CRAB colonization motivate active surveillance and appropriate infection control measures against infected and colonized patients $[3,5,7,8]$.

Conventional methods to screen for CRAB do not sufficiently satisfy the requirements for a robust surveillance tool. Phenotypic methods generally require a few days to determine the antibiotic spectrum, resulting in delays in initiating preventive measures for carriers $[9,10]$. Furthermore, the sensitivity is insufficient to identify colonized patients thoroughly [7, 11]. Molecular methods such as PCR could solve these disadvantages, but these techniques are generally laborious and expensive. Here, we establish a clinical specimendirect Loop-mediated isothermal amplification (LAMP) assay for CRAB as an easier and more costeffective molecular diagnostic system; isothermal amplification finishes within $20 \mathrm{~min}$ and costs are less than 3 U.S. dollars per sample [12]. Since bla $a_{\mathrm{OXA}-23}$ is one of the most prevalent resistance mechanisms among $\mathrm{CRAB}$ in Asia, including Thailand which was 94.2\% in our previous data in Bangkok, we targeted this genotype for our assay development [1, 13-15]. Our clinical specimen-direct LAMP obtains results within $40 \mathrm{~min}$ from sample collection to facilitate early isolation of positive patients. We proved its efficacy as a surveillance tool in an ICU where CRAB is especially problematic [12].

Importantly, active CRAB surveillance has been reported to reduce nosocomial transmission, especially during outbreak situations $[3,16]$, and several guidelines recommended its implementation [5, 17]. It is hypothesized that rapid detection of asymptomatic CRAB carriers enabled medical workers to implement preventive procedures earlier, thus reducing further transmission of CRAB when compared with the conventional culture method. The present study was divided into two arms to compare the incidence rate of transmission; a preintervention period and an intervention period. During the pre-intervention period, preventive measures were implemented following results of the conventional culture method while preventative measures were instituted by the results of LAMP during an intervention period. The primary aim of this study was to analyze whether rapid preventive screening of $\mathrm{CRAB}$ carriers reduces nosocomial transmission rates in ICU settings. We also sought to identify any risk factors associated with CRAB acquisition.

\section{Methods \\ Study settings}

This before and after (quasi-experimental) study was conducted in Mahidol University Faculty of Medicine Ramathibodi Hospital. The study was performed in two discrete medical and surgical ICUs, which had 12 and 15 beds respectively, between December 2013 and January 2015. Conventional culture screening was performed in the first three months (December 2013 to February 2014; pre-intervention period) and the clinical specimen-direct LAMP screening was applied in the subsequent nine months (May 2014 to January 2015; intervention period) following a 2-month wash-out period. All the patients entering the ICUs were screened for $C R A B$ on admission; patients who did not carry $\mathrm{CRAB}$, and those who stayed more than two days, were included due to the definition of nosocomial transmission [18]. The characteristics of the patients were recorded categorically. Circulatory diseased included heart failure, coronary artery disease and etc. Chronic kidney disease (CKD) included all individuals with an estimated glomerular filtration rate of less than $60 \mathrm{ml} / \mathrm{min} / 1.73 \mathrm{~m}^{2}$ on at least 2 occasions 90 days apart. Gastrointestinal disease included ileus, colitis, hemorrhoids, Crohn's disease, and etc. Chronic respiratory disease included chronic obstructive pulmonary disease, asthma, other inflammatory respiratory disorders and etc.

\section{Procedures in the wards}

Surveillance samples were obtained within $24 \mathrm{~h}$ of admission, weekly thereafter and on discharge. Specimens included rectal swabs and, if mechanically ventilated, bronchial aspirates were also obtained since those sites were reported to be appropriate for screening for CRAB $[6,17]$. When either patient sample was positive, we defined the patient as a CRAB carrier. Full-time infection control nurses were assigned to each ward and were engaged in sampling specimens, collecting data, and managing contact precautions. All CRAB carriers were marked with cards to inform medical workers on the necessity of appropriate precautions. Cohorting included the use of a disposable gown and glove as well as isolation of patients using curtains. With the exception of starting time of precautions, we performed the same procedures in both periods. We collected potential confounding factors for all patients. 


\section{Procedures in the laboratory}

All clinical specimens were analyzed using both the conventional culture method and our LAMP assay method. Specimens collected from patients were cultured for at least $18 \mathrm{~h}$ and analyzed by conventional biochemical testing [11] as well as by MALDI-TOF mass spectrometry (Bruker, Leipzig, Germany) [19]. The Sensititre (Thermo Fisher Scientific, Cleveland, $\mathrm{OH}$ ) with the THANF panel of antimicrobial agents was used for the susceptibility tests. Antimicrobial sensitivity testing results (minimum inhibitory concentrations, MICs) were interpreted and reported according to breakpoints published in the CLSI document M100-S23. Subsequently, the same samples were also analyzed by the clinical specimen-direct LAMP as previously reported [12]. Briefly, clinical samples were boiled and centrifuged, then supernatants were used as templates for the LAMP assay. Reactions proceeded at $65^{\circ} \mathrm{C}$ for $20 \mathrm{~min}$.

\section{Statistical analysis}

Data are presented as frequencies with percentages for categorical variables, and median with interquartile range (IQR) for continuous variables. Baseline characteristics between groups were compared using Wilcoxon's rank-sum test or chi-squared test. The primary outcome, which was the rate of $C R A B$ acquisition between each period, was evaluated using Cox proportional hazard model with factors including period, age, sex, intubation, diabetes mellitus, and hypertension. In addition, we performed a multivariable Cox regression with multiple imputation of missing variables. The hazard ratio (HR) was shown with a $95 \%$ confidence interval (CI). As a secondary outcome, we used the multivariable Cox proportional hazard model including factors of medical interventions and antibiotic exposure in the intervention period. Risk factors associated with CRAB carriage on admission were analyzed using multivariable logistic regression since timing did not influence the results. All statistical analyses were performed using $\mathrm{R}$ version 3.4.1 ( $\mathrm{R}$ Foundation for Statistical Computing, Vienna, Austria). All the tests were two-sided, and $p<0.05$ was considered statistically significant.

\section{Results}

\section{Characteristics of study participants during ICU stay}

A total of 318 patients in the pre-intervention period and 1017 patients in the intervention period stayed in either ICU during the study term. Throughout both periods, 172 patients (38 and 134 respectively) were excluded from analysis of nosocomial transmission because they were already colonized with CRAB upon admission. Risk factors for carriage on admission are shown in Table 1. History of hospitalization and history of being a positive carrier were significantly associated with the
Table 1 Risk factors associated with carriage of carbapenemresistant Acinetobacter baumannii (CRAB) on admission to ICUs

\begin{tabular}{lll}
\hline Characteristics & $\begin{array}{l}\text { Odds Ratio } \\
(95 \%(\mathrm{Cl})\end{array}$ & $P$ \\
\hline Female Sex & $1.02(0.68-1.53)$ & 0.93 \\
Age & $1.02(1.00-1.03)$ & 0.78 \\
History of hospitalization within 90 days & $2.85(1.91-4.26)$ & $<0.001$ \\
History of CRAB-positive within 90 days & $60.8(29.7-125)$ & $<0.001$ \\
Circulatory disease & $1.20(0.73-1.97)$ & 0.58 \\
Chronic kidney disease & $1.28(0.78-2.10)$ & 0.38 \\
Gastrointestinal disease & $1.64(0.80-3.37)$ & 0.18 \\
Chronic respiratory disease & $1.35(0.80-2.30)$ & 0.26 \\
Hepatobiliary disease & $0.89(0.32-2.45)$ & 0.82 \\
Cerebral vascular disease & $0.77(0.30-1.96)$ & 0.58 \\
Malignancy (hematologic malignancies and & $0.750 .45-1.27)$ & 0.30 \\
solid tumor) & & \\
Hypertension & $0.85(0.52-1.39)$ & 0.51 \\
Diabetes mellitus & $0.78(0.45-1.34)$ & 0.37 \\
Autoimmune disease & $1.65(0.65-4.16)$ & 0.29 \\
\hline
\end{tabular}

Cl confidence interval

carriage on admission. Patients who stayed less than 2 days, or whose initial screening was not appropriately implemented were excluded from calculation of acquisition rate. Overall, 866 patients (pre-intervention period: 187, intervention period: 679) were analyzed in this study (Fig. 1). We compared characteristics of the participating patients in each term (Table 2). Variables found to be statistically different between the two periods included diabetes mellitus, hypertension, infectious diseases.

\section{Transmission rate of CRAB in ICUs}

A total of 34 and 100 patients obtained CRAB during the study periods, respectively. The transmission rate during ICU stay was 35.2 per 1000 patient-days in the pre-intervention period, compared with 20.9 per 1000 patient-days in the intervention period. The calculated hazard ratio of CRAB transmission was 0.65 (95\% CI, 0.44-0.97) using the multivariable Cox proportional hazard analysis, implying rapid intervention successfully reduced $35 \%$ of nosocomial transmission (unadjusted $P=0.024$, adjusted $P=0.033$ ). Given 68 patients (29 and 39 respectively) had some missing values, we performed multivariable Cox regression with multiple imputation of missing variables. As a result, we could also observe a similar $\mathrm{HR}=0.66[95 \% \mathrm{CI}, 0.44,0.97$; $P=0.034]$. In summary, rapid intervention based on our LAMP assay contributed to reduced nosocomial transmission in ICUs. 

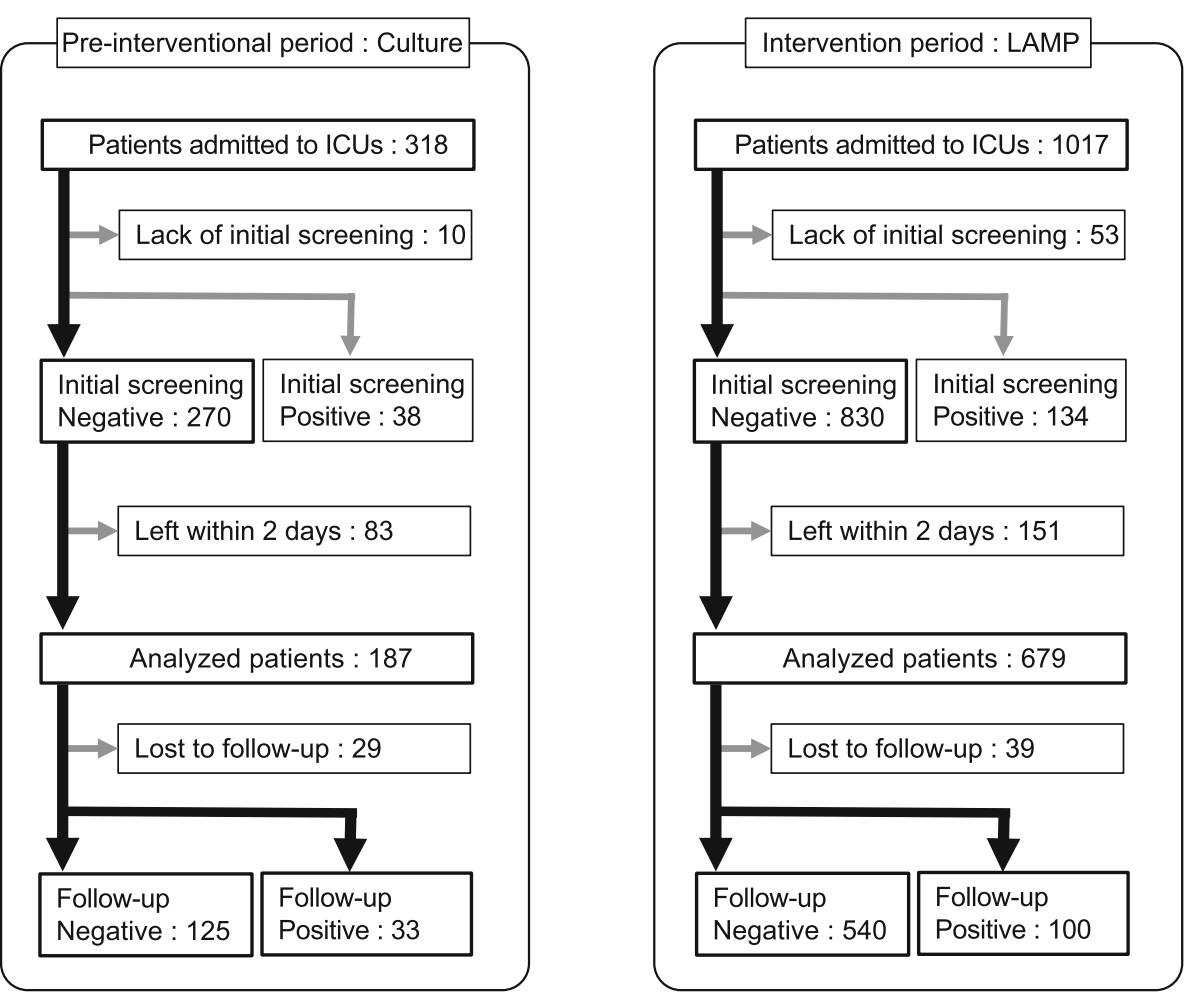

Fig. 1 Flowchart for patient selection in this study

Table 2 Characteristics of patients eligible for this study

\begin{tabular}{|c|c|c|c|c|}
\hline Characteristics & $\begin{array}{l}\text { Pre-interventional Period } \\
(n=187, \%)\end{array}$ & $\begin{array}{l}\text { Interventional Period } \\
(n=679, \%)\end{array}$ & $\begin{array}{l}\text { Total } \\
(n=866, \%)\end{array}$ & $P$ \\
\hline Female Sex & $93(49.7 \%)$ & $315(46.4 \%)$ & $408(47.1 \%)$ & 0.42 \\
\hline Age, median years (IQR) & $66(55,78)$ & $64(54,77)$ & $65(54,77)$ & 0.46 \\
\hline History of hospitalization within 90 days & $57(30.5 \%)$ & $209(30.8 \%)$ & $266(30.7 \%)$ & 0.94 \\
\hline History of CRAB positive within 90 days & $5(2.7 \%)$ & $11(1.6 \%)$ & $16(1.8 \%)$ & 0.34 \\
\hline Duration of ICU stay, median days (IQR) & $5(3.5,9)$ & $6(4,10)$ & $6(4,10)$ & 0.124 \\
\hline Intubation & $132(70.6 \%)$ & $460(67.8 \%)$ & $592(68.4 \%)$ & 0.48 \\
\hline Circulatory disease & $38(20.3 \%)$ & $115(16.9 \%)$ & $153(17.7 \%)$ & 0.28 \\
\hline Chronic kidney disease & $34(18.2 \%)$ & $123(18.1 \%)$ & $157(18.1 \%)$ & 0.98 \\
\hline Gastrointestinal disease & $5(2.7 \%)$ & $43(6.3 \%)$ & $48(5.5 \%)$ & 0.053 \\
\hline Chronic respiratory disease & $28(15.0 \%)$ & $87(12.8 \%)$ & $115(13.3 \%)$ & 0.44 \\
\hline Hepatobiliary disease & $10(5.3 \%)$ & $26(3.8 \%)$ & $36(4.2 \%)$ & 0.36 \\
\hline Cerebral vascular disease & $8(4.3 \%)$ & $55(8.1 \%)$ & $63(7.3 \%)$ & 0.075 \\
\hline Malignancy (hematologic malignancies and solid tumor) & $41(21.9 \%)$ & $188(27.7 \%)$ & $229(26.4 \%)$ & 0.114 \\
\hline Hypertension & $85(45.5 \%)$ & $225(33.1 \%)$ & $310(35.8 \%)$ & 0.002 \\
\hline Diabetes mellitus & $62(33.2 \%)$ & $160(23.6 \%)$ & $222(25.6 \%)$ & 0.008 \\
\hline Autoimmune diseases & $9(4.8 \%)$ & $28(4.1 \%)$ & $37(4.3 \%)$ & 0.68 \\
\hline Infectious diseases & $62(33.2 \%)$ & $167(24.6 \%)$ & $229(26.4 \%)$ & 0.019 \\
\hline
\end{tabular}




\section{Risk factors for CRAB acquisition during ICU stay}

To further confirm risk factors related to acquisition of $\mathrm{CRAB}$, medical interventions being provided as well as antimicrobial exposures were analyzed as possible confounding factors (Table 3). Among all antibiotics, exposure to carbapenems significantly escalated the acquisition rate. Exposure to other antibiotics, as well as all the medical interventions analyzed including intubation, were not associated with CRAB acquisition.

\section{Performance of LAMP assay}

The previously established clinical specimen-direct

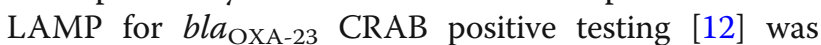
used as a molecular diagnostic assay, and assay performance was evaluated throughout the study period. A total of 2517 clinical samples (tracheal aspirates: 861, rectal swabs; 1656) were obtained, resulting in a sensitivity of 99.1\% (95\% CI, 96.8-99.9\%) and a specificity of $95.0 \%$ (95\% CI, 94.0-95.8\%) (Additional file 1: Table S1), when the gold standard was defined as the results based on culture. Our LAMP assay showed sufficiently high sensitivity and specificity to be used as a screening test in clinical settings.

\section{Discussion}

The issue of CRAB transmission is critical in developing countries because it spreads unnoticed, and appropriate examinations cannot be performed because of a lack of resources [20-22]. The ICUs where this study was carried out were in an endemic situation. Overall, 13.5\% of admitted patients tested positive for CRAB when entering the ICUs, a prevalence comparable with the cohort from Latibeaudiere et al. [6]. Positive patients were more likely to have histories of hospitalization and/ or carriage, although other underlying diseases were not

Table 3 Risk factors associated with carbapenem-resistant Acinetobacter baumannii (CRAB) acquisition during hospitalization

\begin{tabular}{lll}
\hline Characteristics & $\begin{array}{l}\text { Hazard Ratio } \\
(95 \% \mathrm{Cl})\end{array}$ & $p$ \\
\hline Medical intervention & & \\
Intubation & $1.23(0.71-2.15)$ & 0.46 \\
Central venous catheter & $0.69(0.44-1.06)$ & 0.091 \\
Urinary Catheter & $0.69(0.39-1.23)$ & 0.21 \\
Steroid exposure & $0.83(0.53-1.29)$ & 0.41 \\
Antimicrobial exposure & & \\
Carbapenems & $2.54(1.61-4.00)$ & $<0.0001$ \\
Quinolones & $0.73(0.40-1.34)$ & 0.31 \\
Penicillins & $1.34(0.87-2.08)$ & 0.19 \\
3rd and 4th generations of Cephalosporin & $0.69(0.45-1.06)$ & 0.087 \\
Aminoglycosides & $1.59(0.45-5.57)$ & 0.47 \\
\hline
\end{tabular}

Cl confidence interval associated with carriage as shown in Table1. These results were similar to those of other studies $[6,23]$.

Our rapid LAMP assay enabled us to implement earlier intervention for patients carrying CRAB. Using this approach, we achieved a $35 \%$ reduction in $\mathrm{CRAB}$ transmission in the ICUs compared with the use of the conventional culture method (Fig. 2). The difference during each period was in the time period for starting contact precautions. This difference alone contributed to significant reduction of $\mathrm{CRAB}$ nosocomial transmission. Although similar studies targeting methicillin-resistant Staphylococcus aureus have been reported [24-26], to the best of our knowledge clinical trials for $C R A B$ transmission have never been performed.

The contamination rate of $A$. baumannii for environments as well as healthcare workers is higher than other multi-drug resistant organisms (MDROs) [27-31]. Once contaminated, an environment can be a CRAB reservoir for a long period of time since the pathogen can survive for more than several months under dry conditions [32]. Therefore, avoiding contamination is far more important with CRAB than other MDROs. We effectively reduced nosocomial transmission of CRAB in these ICUs because colonized patients were treated properly within a few hours after admission. However, nosocomial transmission could not be eradicated even after we implemented appropriate infection control measures immediately upon identifying a patient as a CRAB carrier. Part of the reason for this is that $A$. baumannii can be acquired directly from the environment, and there are some reports that this bacterium can even be transmitted through aerosols [33, 34]. These contaminated environments could be other origins of transmission.

We performed this study in ICUs where nosocomial transmission was most critical. The average duration of stay in the ICUs is generally shorter than general wards, with many patients leaving within 2 days' duration before culture results can be obtained. In our study, 83 patients in the pre-intervention period and 151 patients in the intervention period left within 2 days, respectively. There were considerable numbers of such carriers among them and they could be generally unnoticed and considered as non-carriers. Using the rapid molecular LAMP assay method, we could implement appropriate precautions earlier than if culture was the only diagnostic test. Therefore, rapid intervention strategies might be more effective in ICU settings than on general wards.

Despite rapid implementation of preventive measures following the use of the LAMP assay, nosocomial transmission still occurred. We considered other potential medical interventions and/or medications that might be associated with acquisition of $\mathrm{CRAB}$, and investigated risk factors for transmission during the intervention period as shown in Table 3. Exposure to carbapenems 


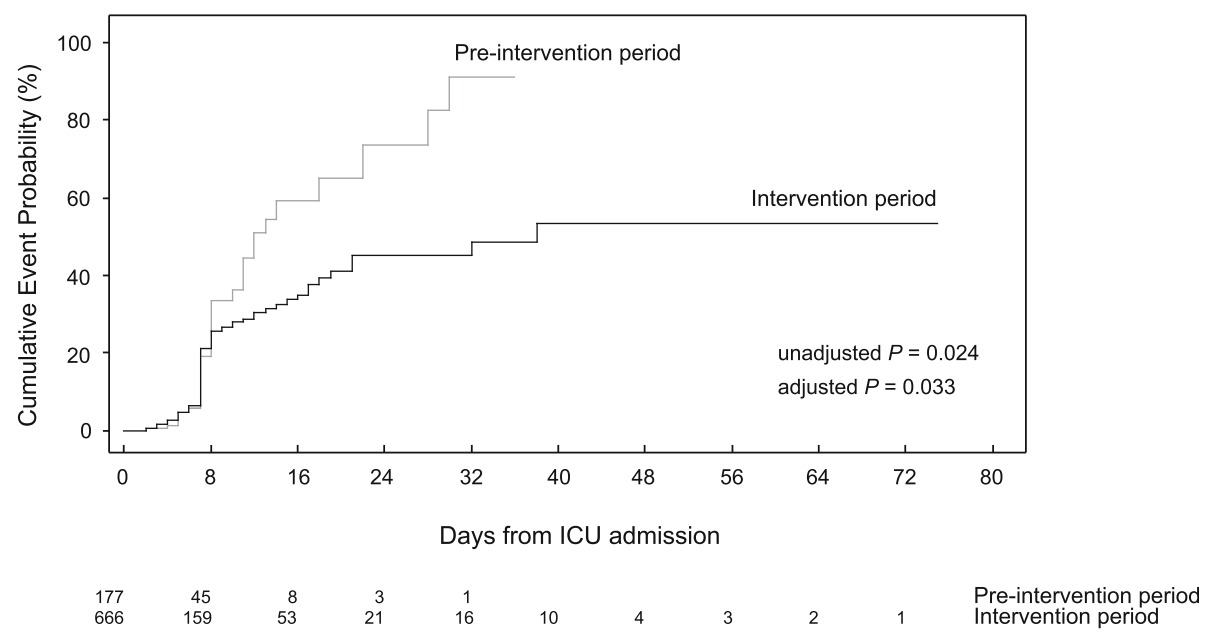

Fig. 2 Kaplan-Meier curves representing cumulative incidence of acquiring carbapenem-resistant Acinetobacter baumannii during ICU stay. The hazard ratio (HR) was calculated by using the multivariable Cox proportional hazard analysis and HR of CRAB transmission was 0.65 (95\% Cl:0.44-0.97) in the interventional period compared with that in the pre-interventional period

was associated with acquisition, a result comparable to other studies [35, 36]. We presume that carbapenems induce a selective pressure for acquisition of CRAB. Intubation, on the other hand, was not recognized as a risk factor in this cohort as other studies also mentioned that mechanical ventilation was not a risk for acquisition despite A. baumannii was major causative organisms of ventilator-associated pneumonia $[5,35]$.

To conduct this study, we adapted the original LAMP assay that targeted $b l a_{\text {OXA-23-type without any DNA }}$ purification steps [12]. Consequently, this assay showed 99.1\% sensitivity, although we targeted this genotype alone. This indicated the diagnostic LAMP test was applicable in clinical settings. Since the mechanism of acquiring drug resistance is becoming complicated, phenotyping methods using culture are often not sufficient for screening. However, PCR, the most popular of genetic analysis tools, is more expensive and laborious than our LAMP assay. The LAMP assay is applicable for such phenotyping experiments and LAMP can be performed without large equipment, demonstrating LAMP testing would be a strong tool to screen for resistant bacteria in resource-limited settings.

Our study could be affected by various factors, such as sample collection procedure, characteristics of ICU and anticipated patients, Hawthorne effect, and various compliances. The biggest bias of this study could be a before-after study design. However, we have compared the patients' backgrounds and there were no significant differences in many variables and we have compensated in categories with significant differences. Furthermore, the pre-intervention period was shorter than the intervention period and was performed during a different period of the seasonal year. Therefore, we would not be able to remove any possible seasonal effect. For example, transmission of Acinetobacter species is more frequent in warm and humid climates [37, 38]. However, since the climate in Bangkok is ideal for A. baumannii throughout the year, the effect of the climate change would be minimized in our study settings. There are some more limitations to our study. First, this study was implemented in particular ICUs within a single center, and the results cannot be applied to any other clinical settings. In particular, the transmission rate was originally higher than the rate in high-income countries [39]. These background factors may impact the result. Second, we implemented this study using our original LAMP assay targeting $b l a_{\text {OXA-23-type alone, although the efficacy had }}$ been confirmed. It could be difficult to perform similar research in other settings. Finally, some patients could not be screened or followed appropriately. This phenomenon was unavoidable, and represented $7.9 \%$, of patients, which is comparable to previous studies [6, 24]. To account for this, we performed the multivariable Cox regression analysis with multiple imputation of missing variables.

In conclusion, we demonstrated the efficacy of using LAMP for rapid CRAB detection, allowing earlier precautions for $C R A B$ carriers to be implemented in clinical settings. Several reports and guidelines recommended active surveillance for halting the spread of MDROs $[3,5,7,16]$. Various novel techniques for bacterial identification are available, and these benefits are reported for treatment of infected patients [40, 41]. This study sheds light on the importance of implementing an immediate active screening test which can prompt earlier action than the conventional method. Rapid diagnostic tests, such as our LAMP assay, will play a critical role in avoiding transmission of CRAB in the future. 


\section{Additional file}

Additional file 1: Additional results of LAMP assay during study period. (DOCX 66 kb)

\section{Abbreviations}

CRAB: Carbapenem-resistant Acinetobacter baumannii; ICU: Intensive care unit; LAMP: Loop-mediated isothermal amplification; MDROs: Multi-drug resistant organisms

\section{Acknowledgments}

We thank all the medical workers in both ICUs for cooperating this study.

\section{Authors' contributions}

NY, SH, YA, PS, KM and KT conceived and designed the study. PS, NC, and SC contributed to all the laboratory works. SS, and KM contributed to clinical work included the data-collection and intervention in the wards. NY, SH, PS, NC, SC, AK, and $\mathrm{KO}$ supported bacterial identification technically. $\mathrm{HH}$ and $\mathrm{KY}$ performed statistical analysis. NY, SH, YA, HH, KY, $H Y, K M$ and $K T$ interpreted the data. NY, SH wrote the initial draft of the manuscript and $\mathrm{YA}, \mathrm{PS}, \mathrm{HH}, \mathrm{HY}, \mathrm{SH}, \mathrm{KO}, \mathrm{KM}$ and $\mathrm{KT}$ revised the draft. All the authors approved the article.

\section{Funding}

This work was supported by JSPS KAKENHI Grant Number 24406022 and J-GRID from the Japan Agency for Medical Research and Development (AMED).

\section{Availability of data and materials}

The datasets used and/or analyzed during the current study are available from the corresponding author on reasonable request.

\section{Ethics approval and consent to participate}

Ethical approval was obtained from the Ethical Clearance Committee on Human Rights Related to Research Involving Human Subjects Faculty of Medicine Ramathibodi Hospital, Mahidol University and the Ethics Committee of Osaka University Graduate School of Medicine. Protocol numbers were ID02-56-09 and 14002, respectively. Because this study was performed as part of routine clinical work, informed consent was omitted as both Institutional Review Boards approved. Instead, patients were informed of the research procedure and wavering rights via a poster in the hospital.

\section{Consent for publication}

All the patients were informed of the research procedure and wavering rights via a poster in the hospital (opt-out)

\section{Competing interests}

The authors declare that they have no competing interests.

\begin{abstract}
Author details
${ }^{1}$ Department of Infection Control and Prevention, Osaka University Graduate School of Medicine, 2-2 Yamadaoka, Suita, Osaka 565-0871, Japan. ${ }^{2}$ Research Institute for Microbial Diseases, Osaka University, Osaka, Japan. ${ }^{3}$ Faculty of Medicine Ramathibodi Hospital, Mahidol University, Bangkok, Thailand. ${ }^{4}$ Department of Medical Statistics, Graduate School of Medicine, Osaka City University, Osaka, Japan. ${ }^{5}$ Faculty of Public Health, Kasetsart University, Sakon Nakhon, Thailand. ${ }^{6}$ Infectious Disease Surveillance Center, National Institute of Infectious Diseases, Tokyo, Japan.
\end{abstract}

Received: 30 January 2019 Accepted: 18 June 2019

Published online: 27 June 2019

\section{References}

1. Peleg AY, Seifert H, Paterson DL. Acinetobacter baumannii: emergence of a successful pathogen. Clin Microbiol Rev. 2008;21(3):538-82.

2. Dijkshoorn L, Nemec A, Seifert H. An increasing threat in hospitals: multidrug-resistant Acinetobacter baumannii. Nat Rev Microbiol. 2007:5(12):939-51.
3. Garnacho-Montero J, Dimopoulos G, Poulakou G, Akova M, Cisneros JM, De Waele J, Petrosillo N, Seifert H, Timsit JF, Vila J, et al. Task force on management and prevention of Acinetobacter baumannii infections in the ICU. Intensive Care Med. 2015;41(12):2057-75.

4. Lemos EV, de la Hoz FP, Einarson TR, McGhan WF, Quevedo E, Castaneda C, Kawai K. Carbapenem resistance and mortality in patients with Acinetobacter baumannii infection: systematic review and meta-analysis. Clin Microbiol Infect. 2014;20(5):416-23.

5. An JH, Kim YH, Moon JE, Jeong JH, Kim SH, Kang SJ, Park KH, Jung SI, Jang HC. Active surveillance for carbapenem-resistant Acinetobacter baumannii in a medical intensive care unit: can it predict and reduce subsequent infections and the use of colistin? Am J Infect Control. 2017;45(6):667-72.

6. Latibeaudiere R, Rosa R, Laowansiri P, Arheart K, Namias N, Munoz-Price LS. Surveillance cultures growing carbapenem-resistant Acinetobacter baumannii predict the development of clinical infections: a retrospective cohort study. Clin Infect Dis. 2015;60(3):415-22.

7. Coyle JR, Kaye KS, Taylor T, Tansek R, Campbell M, Hayakawa K, Marchaim D. Effectiveness and cost of implementing an active surveillance screening policy for Acinetobacter baumannii: a Monte Carlo simulation model. Am J Infect Control. 2014;42(3):283-7.

8. Sydnor ER, Perl TM. Hospital epidemiology and infection control in acutecare settings. Clin Microbiol Rev. 2011;24(1):141-73.

9. Marchaim D, Navon-Venezia S, Leavitt A, Chmelnitsky I, Schwaber MJ, Carmeli Y. Molecular and epidemiologic study of polyclonal outbreaks of multidrug-resistant Acinetobacter baumannii infection in an Israeli hospital. Infect Control Hosp Epidemiol. 2007;28(8):945-50.

10. Marchaim D, Navon-Venezia S, Schwartz D, Tarabeia J, Fefer I, Schwaber MJ, Carmeli Y. Surveillance cultures and duration of carriage of multidrugresistant Acinetobacter baumannii. J Clin Microbiol. 2007;45(5):1551-5.

11. Gerner-Smidt P, Tjernberg I, Ursing J. Reliability of phenotypic tests for identification of Acinetobacter species. J Clin Microbiol. 1991;29(2):277-82.

12. Yamamoto N, Hamaguchi S, Akeda Y, Santanirand P, Kerdsin A, Seki M, Ishii Y, Paveenkittiporn W, Bonomo RA, Oishi K, et al. Clinical specimen-direct LAMP: a useful tool for the surveillance of bla oxa-23-positive carbapenemresistant Acinetobacter baumannii. PLoS One. 2015;10(7):e0133204.

13. Mugnier PD, Poirel L, Naas T, Nordmann P. Worldwide dissemination of the

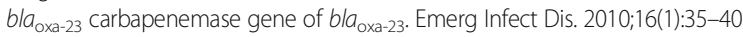

14. Evans BA, Amyes SG. OXA beta-lactamases. Clin Microbiol Rev. 2014;27(2):241-63.

15. Niumsup PR, Boonkerd N, Tansawai U, Tiloklurs M. Carbapenem-resistant Acinetobacter baumannii producing OXA-23 in Thailand. Jpn J Infect Dis. 2009;62(2):152-4.

16. Tacconelli E, Cataldo MA, Dancer SJ, De Angelis G, Falcone M, Frank U, Kahlmeter G, Pan A, Petrosillo N, Rodriguez-Bano J, et al. ESCMID guidelines for the management of the infection control measures to reduce transmission of multidrug-resistant gram-negative bacteria in hospitalized patients. Clin Microbiol Infect. 2014;(20 Suppl):1:1-55.

17. Apisarnthanarak A, Warren DK. Screening for carbapenem-resistant Acinetobacter baumannii colonization sites: an implication for combination of horizontal and vertical approaches. Clin Infect Dis. 2013;56(7):1057-9.

18. Garner JS, Jarvis WR, Emori TG, Horan TC, Hughes JM. CDC definitions for nosocomial infections, 1988. Am J Infect Control. 1988;16(3):128-40.

19. Alvarez-Buylla A, Culebras E, Picazo JJ. Identification of Acinetobacter species: is Bruker biotyper MALDI-TOF mass spectrometry a good alternative to molecular techniques? Infect Genet Evol. 2012;12(2):345-9.

20. Rosenthal VD, Bijie H, Maki DG, Mehta Y, Apisarnthanarak A, Medeiros EA, Leblebicioglu H, Fisher D, Alvarez-Moreno C, Khader IA, et al. International nosocomial infection control consortium (INICC) report, data summary of 36 countries, for 2004-2009. Am J Infect Control. 2012;40(5):396-407.

21. Lim C, Takahashi E, Hongsuwan M, Wuthiekanun V, Thamlikitkul V, Hinjoy S, Day NP, Peacock SJ, Limmathurotsakul D. Epidemiology and burden of multidrug-resistant bacterial infection in a developing country. Elife. 2016;5.

22. Hsu LY, Apisarnthanarak A, Khan E, Suwantarat N, Ghafur A, Tambyah PA. Carbapenem-resistant Acinetobacter baumannii and Enterobacteriaceae in south and Southeast Asia. Clin Microbiol Rev. 2017;30(1):1-22.

23. Blanco N, Harris AD, Rock C, Johnson JK, Pineles L, Bonomo RA, Srinivasan A, Pettigrew MM, Thom KA. Risk factors and outcomes associated with multidrug-resistant Acinetobacter baumannii upon intensive care unit admission. Antimicrob Agents Chemother. 2018;62(1).

24. Jeyaratnam D, Whitty CJ, Phillips K, Liu D, Orezzi C, Ajoku U, French GL. Impact of rapid screening tests on acquisition of meticillin resistant 
Staphylococcus aureus: cluster randomised crossover trial. BMJ. 2008; 336(7650):927-30.

25. Cunningham R, Jenks $P$, Northwood J, Wallis M, Ferguson $S$, Hunt $S$. Effect on MRSA transmission of rapid PCR testing of patients admitted to critical care. J Hosp Infect. 2007;65(1):24-8.

26. Harbarth S, Masuet-Aumatell C, Schrenzel J, Francois P, Akakpo C, Renzi G Pugin J, Ricou B, Pittet D. Evaluation of rapid screening and pre-emptive contact isolation for detecting and controlling methicillin-resistant Staphylococcus aureus in critical care: an interventional cohort study Crit Care. 2006;10(1):R25.

27. Morgan DJ, Rogawski E, Thom KA, Johnson JK, Perencevich EN, Shardell M Leekha S, Harris AD. Transfer of multidrug-resistant bacteria to healthcare workers' gloves and gowns after patient contact increases with environmental contamination. Crit Care Med. 2012;40(4):1045-51.

28. Rosa R, Arheart KL, Depascale D, Cleary T, Kett DH, Namias N, Pizano L, Fajardo-Aquino Y, Munoz-Price LS. Environmental exposure to carbapenemresistant Acinetobacter baumannii as a risk factor for patient acquisition of $A$. baumannii. Infect Control Hosp Epidemiol. 2014;35(4):430-3.

29. Playford EG, Craig JC, Iredell JR. Carbapenem-resistant Acinetobacter baumannii in intensive care unit patients: risk factors for acquisition, infection and their consequences. J Hosp Infect. 2007;65(3):204-11.

30. Jawad A, Seifert H, Snelling AM, Heritage J, Hawkey PM. Survival of Acinetobacter baumannii on dry surfaces: comparison of outbreak and sporadic isolates. J Clin Microbiol. 1998;36(7):1938-41.

31. Thom KA, Rock C, Jackson SS, Johnson JK, Srinivasan A, Magder LS, Roghmann MC, Bonomo RA, Harris AD. Factors leading to transmission risk of Acinetobacter baumannii. Crit Care Med. 2017;45(7):e633-9.

32. Chemaly RF, Simmons S, Dale C Jr, Ghantoji SS, Rodriguez M, Gubb J, Stachowiak J, Stibich M. The role of the healthcare environment in the spread of multidrug-resistant organisms: update on current best practices for containment. Ther Adv Infect Dis. 2014;2(3-4):79-90.

33. Yakupogullari $Y$, Otlu B, Ersoy Y, Kuzucu C, Bayindir Y, Kayabas U, Togal T, Kizilkaya C. Is airborne transmission of Acinetobacter baumannii possible: a prospective molecular epidemiologic study in a tertiary care hospital. Am J Infect Control. 2016;44(12):1595-9.

34. Munoz-Price LS, Fajardo-Aquino Y, Arheart KL, Cleary T, DePascale D, Pizano L, Namias N, Rivera JI, O'Hara JA, Doi Y. Aerosolization of Acinetobacter baumannii in a trauma ICU*. Crit Care Med. 2013;41(8):1915-8.

35. Munoz-Price LS, Rosa R, Castro JG, Laowansiri P, Latibeaudiere R, Namias N, Tarima S. Evaluating the impact of antibiotic exposures as time-dependent variables on the acquisition of carbapenem-resistant Acinetobacter baumannii. Crit Care Med. 2016;44(10):e949-56.

36. Castelo Branco Fortaleza CM, Moreira de Freitas F, da Paz Lauterbach G. Colonization pressure and risk factors for acquisition of imipenem-resistant Acinetobacter baumannii in a medical surgical intensive care unit in Brazil. Am J Infect Control. 2013;41(3):263-5.

37. Smith PW. Seasonal incidence of Acinetobacter infection. J Infect Dis. 1979; 140(2):275-6.

38. McDonald LC, Banerjee SN, Jarvis WR. Seasonal variation of Acinetobacter infections: 1987-1996. Nosocomial Infections Surveillance System Clin Infect Dis. 1999;29(5):1133-7.

39. Allegranzi B, Bagheri Nejad S, Combescure C, Graafmans W, Attar H, Donaldson L, Pittet D. Burden of endemic health-care-associated infection in developing countries: systematic review and meta-analysis. Lancet. 2011;377(9761):228-41.

40. Banerjee R, Teng CB, Cunningham SA, Ihde SM, Steckelberg JM, Moriarty JP, Shah ND, Mandrekar JN, Patel R. Randomized trial of rapid multiplex polymerase chain reaction-based blood culture identification and susceptibility testing. Clin Infect Dis. 2015;61(7):1071-80

41. Huang AM, Newton D, Kunapuli A, Gandhi TN, Washer LL, Isip J, Collins CD, Nagel JL. Impact of rapid organism identification via matrix-assisted laser desorption/ionization time-of-flight combined with antimicrobial stewardship team intervention in adult patients with bacteremia and candidemia. Clin Infect Dis. 2013;57(9):1237-45.

\section{Publisher's Note}

Springer Nature remains neutral with regard to jurisdictional claims in published maps and institutional affiliations.

\section{Ready to submit your research? Choose BMC and benefit from:}

- fast, convenient online submission

- thorough peer review by experienced researchers in your field

- rapid publication on acceptance

- support for research data, including large and complex data types

- gold Open Access which fosters wider collaboration and increased citations

- maximum visibility for your research: over $100 \mathrm{M}$ website views per year

At BMC, research is always in progress.

Learn more biomedcentral.com/submissions 